\title{
An injectable self-healing coordinative hydrogel with antibacterial and angiogenic properties for diabetic skin wound repair
}

\author{
Hao Chen ${ }^{1,2}$, Ruoyu Cheng ${ }^{1}$, Xin Zhao ${ }^{3}$, Yuhui Zhang ${ }^{2}$, Allison Tam³ ${ }^{3}$ Yufei Yan ${ }^{1}$, Haokai Shen ${ }^{4}$, Yu Shrike Zhang ${ }^{5}$, \\ Jin Qi ${ }^{1}$, Yonghai Feng ${ }^{6}$, Lei Liu', Guoging Pan $\mathbb{1}^{6}$, Wenguo Cui (iD) and Lianfu Deng ${ }^{1}$
}

\begin{abstract}
We report here an injectable, self-healing coordinative hydrogel with antibacterial and angiogenic properties for diabetic wound regeneration. The hydrogel was prepared by coordinative cross-linking of multi-arm thiolated polyethylene glycol (SH-PEG) with silver nitrate (AgNO3). Due to the dynamic nature of Ag-S coordination bond and bacteria-killing activity of $\mathrm{Ag}+$, the resultant coordinative hydrogel featured self-healing, injectable and antibacterial properties. In this study, we synchronously loaded an angiogenic drug, desferrioxamine (DFO), in the coordinative hydrogel during cross-linking. We finally obtained a multifunctional hydrogel that is manageable, resistant to mechanical irritation, antibacterial and angiogenic in vitro. Our in vivo studies further demonstrated that the injectable self-healing hydrogel could efficiently repair diabetic skin wounds with low bacteria-infection and enhance angiogenic activity. In short, besides diabetic skin wound repair, such dynamic multifunctional hydrogel scaffolds would show great promise in the regeneration of different types of exposed wounds, in particular, in situations with disturbed physiological functions, high risk of bacterial infections, and external mechanical irritation.
\end{abstract}

\section{Introduction}

Diabetes can lead to nonhealing chronic ulcers over tendons, bones, and joints, and such conditions have to date led to more than 20 million patients suffering a single leg amputation. It is believed that the number of patients who will require lower limb amputation annually will double by $2030^{1}$. The primary cause of the dreaded and chronic diabetic ulcer complication is impaired vessel formation, particularly microvasculature formation, which is critical for the delivery of oxygen, nutrients, and growth

Correspondence: Guoqing Pan (panguoqing@ujs.edu.cn) or

Wenguo Cui (wgcui80@hotmail.com) or Lianfu Deng (If_deng@126.com)

${ }^{1}$ Shanghai Key Laboratory for Prevention and Treatment of Bone and Joint

Diseases, Shanghai Institute of Traumatology and Orthopaedics, Ruijin Hospital, Shanghai Jiao Tong University School of Medicine, 197 Ruijin 2nd Road,

200025 Shanghai, China

${ }^{2}$ Department of Spinal Surgery, Renji Hospital, Shanghai Jiao Tong University

School of Medicine, 160 Pujian Road, 200120 Shanghai, China

Full list of author information is available at the end of the article.

These authors contributed equally: Hao Chen, Ruoyu Cheng, Xin Zhao factors, all of which are needed for wound healing, especially in the early stages ${ }^{2,3}$. Without sufficient angiogenesis (the formation of capillary blood vessel networks), high levels of glucose accumulate at the wound site, leading to ischemia and tissue necrosis ${ }^{4,5}$. Thus, the reestablishment of the vascular network of diabetic wounds in the early stages of healing is essential to prevent wound expansion and ulcer formation in diabetic patients.

Another issue that adversely affects diabetic wound healing is bacterial infection. In clinical settings, the severity of a diabetic foot infection can range from superficial paronychia to life-threatening bone infections ${ }^{6}$. Bacterial infection is also associated with the increased frequency and length of hospitalization and the risk of extreme lower limb amputation ${ }^{7}$. It is well-known that wounds of an external origin, especially skin ulcers, are more likely to come in contact with exogenous microorganisms, increasing the probability of bacterial 


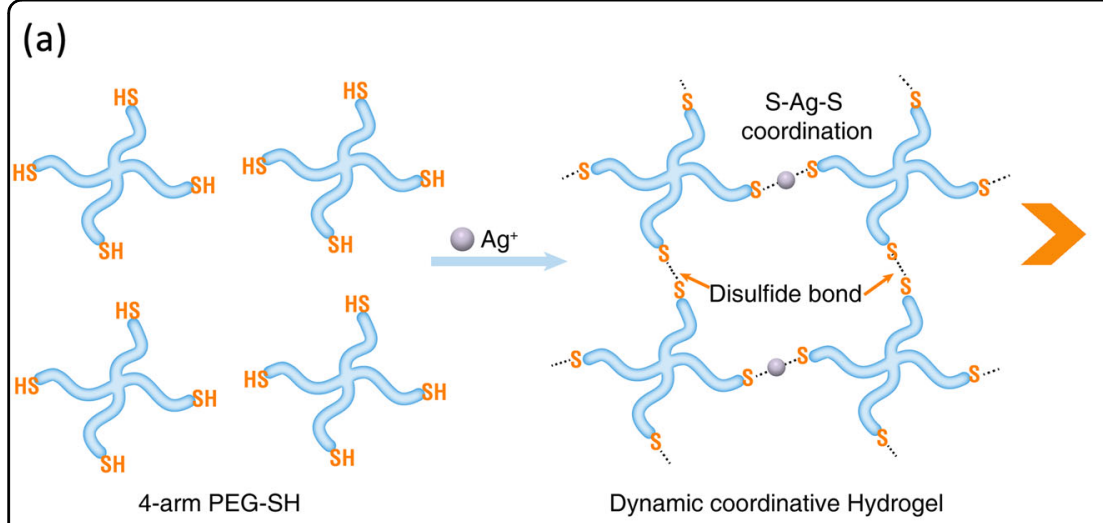

(d)

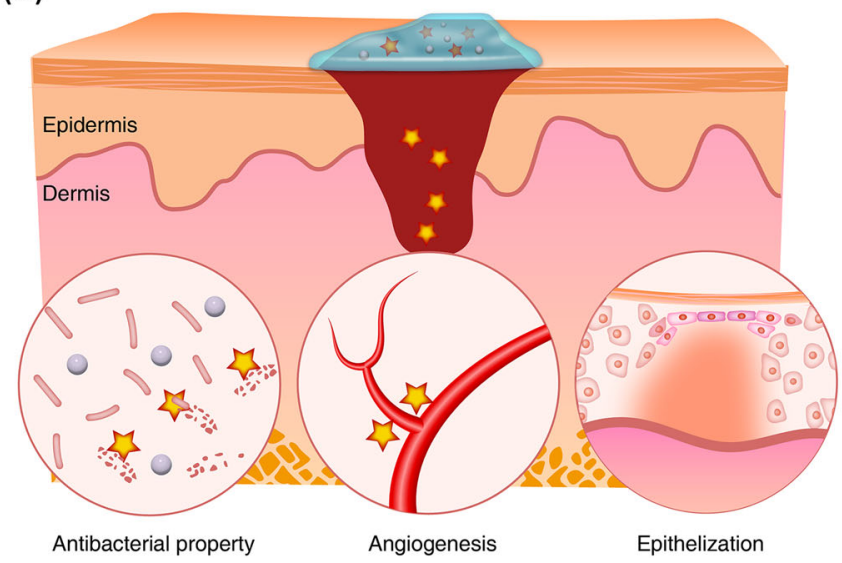

(b)

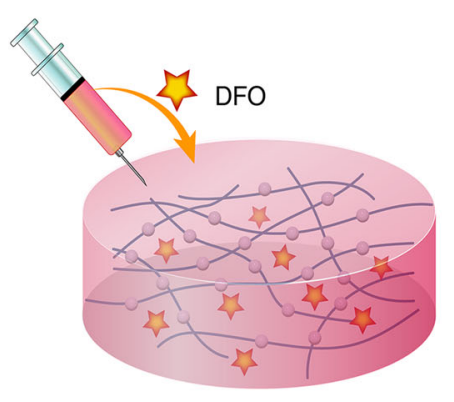

(c)

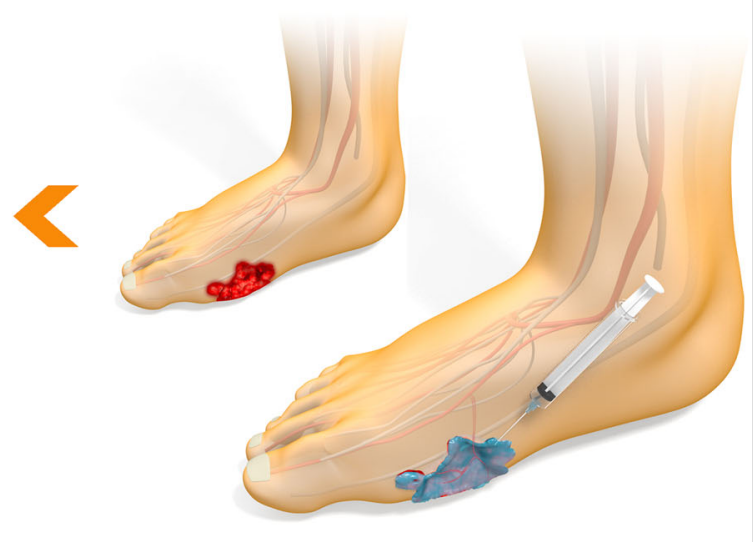

Scheme 1 a Schematic illustration of the self-healing Ag(I)-thiol (Au-S) coordinative hydrogel developed by mixing 4-arm-PEG-SH with $\mathrm{AgNO}_{3}$. b In situ encapsulation of drug (DFO) to obtain an injectable, self-healing, antibacterial, and angiogenic multifunctional hydrogel for diabetic skin wound repair. c Foot ulcers of type I diabetes (left) and therapeutic effect after hydrogel treatment (right). d Mechanism of the hydrogel in repairing skin defects through injection

infections. The impaired leukocyte function of diabetic patients results in metabolic abnormalities, which commonly lead to inadequate neutrophil and macrophage migration to wounds ${ }^{8}$. Furthermore, this impairment predisposes diabetics to higher risk of wounds and to the danger of chronic diabetic diseases such as angiopathy'. Hence, better methods for the prevention of bacterial infections during the treatment of diabetic skin ulcers are highly sought after.

The early clinical treatments of diabetic wounds are mainly based on the use of protective dressings with antibiotics or subsidiary drugs ${ }^{10}$. To improve the therapeutic effects of such approaches in the treatment of diabetic wounds, there has been a growing interest in the development of three-dimensional bioengineered substitutes in the form of films, sponges, microfibers, and nanofibers with desired functions such as angiogenesis ${ }^{11}$. For example, we have previously demonstrated that electrospun nanofibrous and porous hydrogel scaffolds loaded with angiogenic factors could efficiently improve diabetic skin regeneration ${ }^{4,5}$. Undoubtedly, these scaffolds, which possess a similar structure and biochemistry to the natural extracellular matrix (ECM), ensure skin tissue regeneration when combined with biomolecules. However, the current scaffolds are based on monofunctional polyester fibers or crosslinked hydrogels and must be shaped into membrane-like structures prior to implantation. The function of a scaffold would be significantly reduced if the scaffold were to fail under the application of external stresses, and therefore, advanced scaffolds for diabetic wound treatment should feature multiple functions such as the promotion of early-stage vascular formation and the prevention of bacterial infections. More importantly, these advanced scaffolds should be able to resist mechanical irritation, which is highly inevitable in diabetic skin wound repair.

Herein, we report an injectable, self-healing coordinative hydrogel with antibacterial and angiogenic properties for diabetic wound regeneration. The hydrogel (referred to as Ag-SH-PEG) was simply prepared using 
coordinative crosslinking of multi-arm thiolated polyethylene glycol (SH-PEG) with silver nitrate $\left(\mathrm{AgNO}_{3}\right)$ (Scheme 1a). Due to the dynamic and reversible nature of the $\mathrm{Ag}-\mathrm{S}$ coordination bond, the resultant coordinative hydrogel featured self-healing properties after repeated rupture and injectable properties when applied through a medical needle ${ }^{12}$. Such self-healing and injectable properties are particularly appealing for skin wound repair because they help reduce gel fragmentation and integrate ruptured gels at the target site, even after external mechanical destruction, and hence can continuously support skin wound healing ${ }^{13}$. Moreover, the hydrogel network gradually releases antibacterial silver ions, which are highly attractive for use in susceptible open diabetic skin wounds. Due to the incorporation of an angiogenic drug, desferrioxamine (DFO), into the coordinative hydrogel, we finally obtained a multifunctional hydrogel that is manageable, resistant to external stress, antibacterial, and angiogenic (Scheme $1 b)^{14}$. As an example, an irregular wound resulting from a foot ulcer is shown; in such cases, it is typically difficult for the vessels to grow in (Scheme 1c), which may be overcome in our proposed formulation. Furthermore, no self-healing hydrogels have been reported to include intrinsic structural properties that promote angiogenesis while simultaneously preventing bacterial infections ${ }^{15-17}$. We anticipate that such unique multifunctional hydrogels will exhibit efficient anti-infective abilities, enhance angiogenic activity, and subsequently accelerate tissue healing in diabetic skin wound sites (Scheme 1d).

\section{Materials and methods}

\section{Ag-based hydrogel preparation}

To prepare the hydrogels, 4-arm-PEG-SH $(50 \mathrm{mg}$, Mw: 20,000, purchased from Shanghai Zhenzhun Biological Technology Co., Ltd) was dissolved in water $(250 \mu \mathrm{L})$. Then, $100 \mu \mathrm{L}$ of an aqueous solution of $\mathrm{AgNO}_{3}(0.1 \mathrm{M})$ was diluted in $150 \mu \mathrm{L}$ of deionized water. Each aqueous solution was placed in two separate tubes. Upon mixing, the two solutions formed transparent gels after a few seconds. The DFO-loaded hydrogel was similarly prepared except that the 4-arm-PEG-SH $(50 \mathrm{mg})$ was dissolved in the DFO solution $(20 \mathrm{mg} / \mathrm{mL})$.

\section{Mechanical and self-healing properties}

To examine the hydrogel's self-healing properties, strain-dependent oscillatory measurements were conducted on the hydrogels to determine the critical strain value required to disrupt the gel network and cause the transition to a solution state. Stress-strain measurements were established by subjecting hydrogels to a low strain $(\gamma=0.05 \%)$ during the first $100 \mathrm{~s}$, followed by a high strain $(\gamma=500 \%)$ during the next $50 \mathrm{~s}$. When high strain was discontinued, a low magnitude strain $(\gamma=0.05 \%)$ was applied. A digital camera was used to capture the process of self-healing and injection at the macroscopic level. To demonstrate their self-healing ability, the hydrogels were divided into two parts, and then the two segments were placed within surface contact for $15 \mathrm{~min}$. In addition, rhodamine-dyed hydrogels were injected using a $1-\mathrm{mL}$ syringe with needles as small as $0.5 \mathrm{~mm}$ in diameter to investigate the injectability of the hydrogels.

\section{In vitro antibacterial activity}

In this study, the antimicrobial efficacy of the hydrogels was tested against Staphylococcus aureus. First, the microorganisms were sub-cultured in appropriate culture media to confirm their purity. Aliquots of the suspension Staphylococcus aureus-containing were spread on the agar surface for inoculation. Then, $200 \mu \mathrm{L}$ of hydrogel was placed at the center of the plate, and the plate was incubated for $120 \mathrm{~h}$ consistently at $37^{\circ} \mathrm{C}$. The inhibition zones were measured at 24,72 , and $120 \mathrm{~h}$. The diameters of the growth inhibition zones were analyzed statistically using one-way analysis of variance (ANOVA) and an unpaired $t$-test.

\section{In vitro cell biocompatibility measurements}

Human umbilical vein endothelial cells (HUVECs) were used to investigate cell adhesion and proliferation on the hydrogels. A Live/Dead ${ }^{\oplus}$ assay was carried out to test the viability of the cells. The cells were seeded on the surface of the hydrogels at a density of $1 \times 10^{5}$ cells $/ \mathrm{mL}$. At designated time points, cell viability was tested according to the manufacturer's instruction using the Live/Dead assay (Invitrogen, USA). The stained samples were visualized using a confocal laser scanning microscope (LSM800, ZEISS, Germany). Cell cytotoxicity was analyzed with the Cell Counting Kit-8 (CCK-8) assay. The cell seeding and culture protocols were the same as above. Briefly, $0.5 \mathrm{~mL}$ of fresh Dulbecco's Modified Eagle Medium (DMEM, Gibco, USA) containing 10\% CCK-8 solution (Dojindo Kagaku, Japan) was added to each well. After $2 \mathrm{~h}, 100 \mu \mathrm{L}$ of the mixed medium was transferred to a 96-well plate. The solution absorbance was measured at a wavelength of $450 \mathrm{~nm}$ with a microplate reader (Infinite F50, TECAN, Switzerland). All studies were performed in triplicate.

\section{In vitro tube-formation assay}

Coverslips for cell culture were prepositioned in the 24well plate. Hydrogels and DFO-loaded hydrogels were laid over the coverslips, while the control group was coated with growth factor-reduced Matrigel. HUVECs were seeded $\left(6 \times 10^{4}\right.$ cells/well $)$ and incubated with $5 \% \mathrm{CO}_{2}$ at $37^{\circ} \mathrm{C}$ for $12 \mathrm{~h}$. The HUVECs were then rinsed three times with phosphate-buffered saline (PBS, $10 \mathrm{~min}$ each) and fixed with $4 \%$ polyformaldehyde for $15 \mathrm{~min}$. The fixed cells were permeabilized with $0.2 \%$ Triton X-100 and then 
incubated with $1 \%$ BSA for 30 min to block nonspecific antibody binding. Cells were stained with fluorescein isothiocyanate (FITC)-phalloidin-PBS solution $(200 \mu \mathrm{L}$ $1 \times$ PBS containing $5 \mu \mathrm{L}$ FITC-phalloidin) in the dark at room temperature for $1 \mathrm{~h}$, followed by Hoechst 33258 (10 $\mathrm{mg} / \mathrm{mL}$ ) staining for $15 \mathrm{~min}$. Fluorescence images of 5 randomly selected fields were visualized under a $4 \times$ fluorescence microscope (Imager M1, ZEISS, Germany). ImageJ software was used to measure tube lengths and obtain an average value.

\section{Induction of Type I diabetic rat models}

All animal feeding and experimental contents were approved by the Animal Ethics Committee of Ruijin Hospital, Shanghai Jiaotong University, School of Medicine. Eight-week-old Sprague-Durer rats (SD rats) were purchased from Shanghai SLAC Experimental Animals, Inc. Rats were housed 3 per cage and fed ad libitum with the temperature of the Animal Ward controlled at $22 \pm 2{ }^{\circ} \mathrm{C}$. After 2 weeks of acclimatization, rats weighting 300-350 g were randomly selected for the induction of type I diabetes. The prematched STZ solution was given by intraperitoneal injection of $70 \mathrm{mg} / \mathrm{kg}$. The blood glucose levels of the rats were measured weekly through the tail vein. Rats whose blood glucose levels remained greater than $300 \mathrm{mg} / \mathrm{dL}$ for 6 weeks were identified as successful type I diabetic rats.

\section{Skin defect of type I diabetic rats}

The experiment was divided into three groups: the control group, the hydrogel group, and the DFO-loaded hydrogel group. A total of 48 type I diabetic rats were randomly selected to receive general anesthesia by intraperitoneal injection of $2.5 \%$ pentobarbital sodium at a volume of $0.3 \mathrm{~mL} / 100 \mathrm{~g}$. The rats were fixed and then shaved with an animal electric shaver to remove the hair on their backs. Further hair removal was achieved with irritation-free depilation cream to fully expose the operative area. Alcohol (70\%) was used to disinfect the surgical area, and then, a sterile towel was applied. A 15$\mathrm{mm}$-diameter circular full skin defect was drilled with a corneal trephine on both sides of the midline of the back until the depth reached the sarcolemma. Either the hydrogel or DFO-loaded hydrogel solution was dripped onto the wound surface with a $5-\mathrm{mL}$ syringe until the height of the solution dripped was at the same level as that of the normal skin, and the hydrogel solution was in situ crosslinked. The same skin lesion without intervention was established as the control group. The wounds of the three groups of rats were covered with $3 \mathrm{M}$ breathable patches, each of which was then covered with a sterile gauze pad to prevent the rats from biting. All operations were performed under aseptic conditions. The rats were fed alone after operation. The rats were photographed by a parameter-fixed SLR camera at the same height on days $0,4,7,10$ and 14 post-operation. ImageJ was used to measure and calculate the circumference of each wound boundary to obtain macroscopic wound healing data.

\section{H\&E staining}

To avoid inaccurate results caused by skin contraction, complete specimens of the wounds and the normal skin tissue within $3 \mathrm{~mm}$ around the wounds were collected on days $4,7,10$, and 14 post-operation. The specimens were fixed overnight using $4 \%$ polyoxymethylene and then dehydrated by an automatic dehydrator. After embedding in paraffin, the specimens were cut into $5-\mu \mathrm{m}$ slices for hematoxylin and eosin (H\&E) staining. The creeping distance of the newborn epithelium was taken as an objective index to observe skin healing. The images were taken with a light microscope (Imager M1, ZEISS, Germany). ImageJ was used to measure the creeping distance at each time point, and then, statistical analyses were performed.

\section{Immunohistochemical analysis}

For immunostaining, skin sections were air-dried, permeabilized for $10 \mathrm{~min}$ in $0.3 \%$ Triton X-100, blocked in $5 \%$ goat serum at room temperature for $30 \mathrm{~min}$, and probed with the primary antibody diluted in $5 \%$ goat serum with PBS overnight at $4{ }^{\circ} \mathrm{C}$. The primary anti-Staphylococcus aureus antibody (ab68950, Abcam, 1:200) and anti-CD31 antibody (NB100-2284, Novus, 1:200) were used. After primary antibody incubation, the sections were washed with PBS and incubated with the appropriate Alexa Fluor-coupled secondary antibodies (Molecular Probes, Life Technologies, USA, 1:400) for $1 \mathrm{~h}$ at room temperature. Nuclei were counterstained with 4 , 6-Diamidino-2-phenyindole dilactate (DAPI, Life Technologies, USA). Finally, coverslips were sealed with nail polish, and the sections were analyzed under a confocal laser scanning microscope. Signal intensities were quantified using ImageJ software. All studies were performed in triplicate.

\section{Statistical analysis}

All obtained data were expressed as the mean \pm standard deviation (SD). Statistical analysis was performed using the SPSS 19.0 software package (SPSS, IBM, USA). The statistical significance of the difference was measured using one-way analysis of variance (ANOVA) and Student's $t$-test $\left({ }^{*} P<0.05,{ }^{* *} P<0.01\right.$, and $\left.{ }^{* * *} P<0.001\right)$. $P<0.05$ was considered statistically significant.

\section{Results and discussion}

The ideal hydrogel for skin repair must not only be able to endure external strain but must also exhibit selfhealing properties before its drug-loading capabilities can 

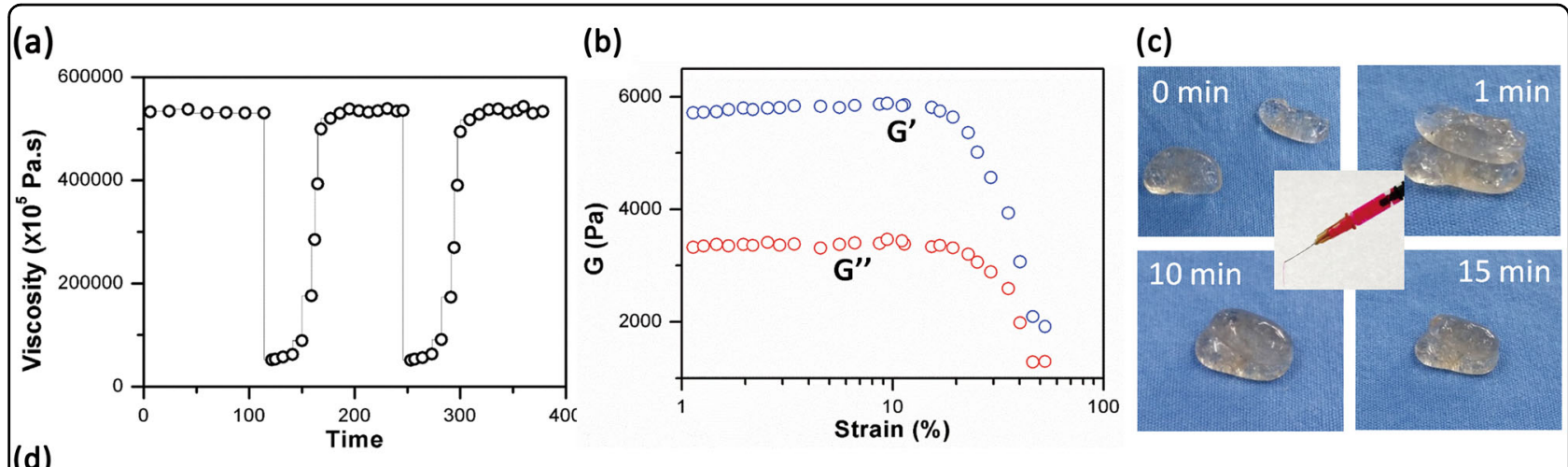

(d)
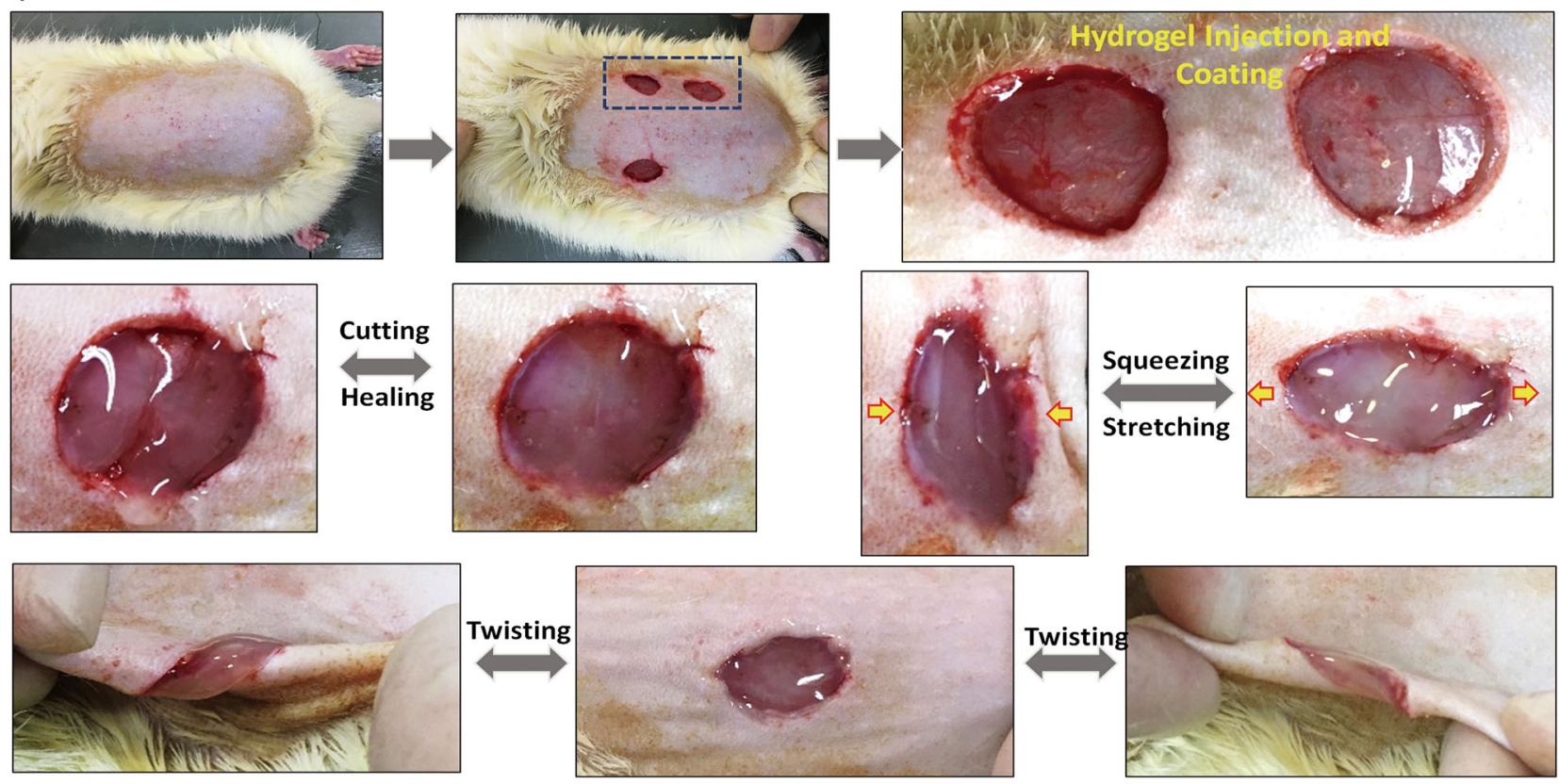

Fig. 1 Mechanical and self-healing properties of the Ag-SH-PEG hydrogels. a Measure of viscosity parameters in relation to time in seconds. Strain shearing rate alternated between $0.1 \%$ strain for $50 \mathrm{~s}$ and $1000 \%$ strain for $100 \mathrm{~s}$. b Strain sweep measurements of the storage moduli $\left(\mathrm{G}^{\prime}\right.$ for elastic modulus and $\mathrm{G}^{\prime \prime}$ for loss modulus), measured in $\mathrm{Pa}$, on the strain of the hydrogels (\%) to determine the tensile strength. $\mathbf{c}$ Images of a hydrogel sample cut in half exhibiting the self-healing property, taken at 0,1, 10, and 15 min. $\mathbf{d}$ SD rats with full-thickness wounds (diameters $15 \mathrm{~mm}$ with error within $1 \mathrm{~mm}$ ), followed by injection of the hydrogels. The hydrogels retained their shapes despite being cut, squeezed, stretched, and twisted

be considered for therapeutic purposes. Fortunately, explorations of dynamic covalent bonds, including metal-thiol coordination, reversible boronate-diol, and benzoic-imine interactions, have greatly facilitated the development of dynamic hydrogels with self-healing and injectable properties ${ }^{12,18-22}$. In this work, we employed the antibacterial $\mathrm{Ag}^{+}$and biocompatible 4-arm-PEG-SH to fabricate a Ag-SH coordinative dynamic hydrogel for skin wound repair. The structure of the hydrogel was investigated by Fourier-transform infrared spectroscopy (FTIR) to explore the mechanism used in hydrogel formation. Both the 4-arm-PEG-SH and the hydrogel were examined, as shown in Figure S1. Usually, the characteristic peaks of the disulfide bond are observed in the range of $500-600 \mathrm{~cm}^{-1}$; however, in this work, to highlight the function of coordination in the formation of the hydrogel network, the disulfide bonds were not the focus. The hydrogel was formed predominantly based on two mechanisms, disulfide bond formation and coordination between $\mathrm{Ag}$ and $\mathrm{S}$, as shown in the following formula:

$2 \mathrm{Ag}^{+}+2 \mathrm{SH}-\mathrm{PEG} \rightarrow 2 \mathrm{Ag}+\mathrm{PEG}-\mathrm{S}-\mathrm{S}-\mathrm{PEG}+2 \mathrm{H}^{+}$

$\mathrm{Ag}+2 \mathrm{SH}-\mathrm{PEG} \rightarrow(\mathrm{PEG}-\mathrm{SH}) \ldots \mathrm{Ag} \ldots(\mathrm{PEG}-\mathrm{SH})$

For skin diabetic wounds, the ability to inject a malleable hydrogel that is capable of fusing itself back together 
in the event of damages holds significant value in the preservation of gel function and form. Thus, before examining additional characteristics, it was necessary to assess the mechanical and self-healing properties of the synthesized DFO-hydrogels following injection. The rheological properties were characterized with a rheometer by measuring the changes in viscosity ${ }^{23}$. Fig. 1a illustrates the hydrogel's capability to recover its structure under shear stress in a thixotropy test ${ }^{24}$. The hydrogel's viscosity was consistently recorded at an average value of $532,342 \pm 2,616$ at a low shear rate and 63,056 $\pm 15,113$ at a high shear rate, indicating that the hydrogel's structure had a high recovery rate and could withstand large shear stresses. This is further illustrated in Fig. 1b, where both the elastic modulus $\left(G^{\prime}\right)$ and loss modulus $\left(G^{\prime \prime}\right)$ could withstand an increase in strain of $\sim 50 \%$. Specifically, the elastic modulus $\left(\mathrm{G}^{\prime}\right)$ of the hydrogel was $\sim 5700 \mathrm{~Pa}$, and the loss modulus $\left(\mathrm{G}^{\prime \prime}\right)$ was $\sim 3300 \mathrm{~Pa}$. Fig. 1b displays the results of a strain amplitude sweep. Our results showed that at low strain (corresponding to before injection), $G^{\prime}$ $(6000 \mathrm{~Pa})$ was greater $\mathrm{G}^{\prime \prime}(3200 \mathrm{~Pa})$. Moreover, both $\mathrm{G}^{\prime}$ and $\mathrm{G}^{\prime \prime}$ were on the same order of magnitude, which indicated a gel-like material state rather than a solid state. If a material were in a solid state, then $G^{\prime}$ would be several orders of magnitude larger than $G^{\prime \prime}$. When the strain increased (corresponding to the injection process), $G^{\prime}$ decreased significantly and became less than $G^{\prime \prime}\left(G^{\prime}<G^{\prime \prime}\right)$, suggesting that the material would exist in a liquid state (i.e., injectable). As shown in Fig. 1c, the separated fragments of the post-incision hydrogel fused within $15 \mathrm{~min}$ at room temperature, thus returning the hydrogel to its original state and displaying a self-healing property. Moreover, the flexibility in the shape of the hydrogel allowed for injections with needles as small as $0.5 \mathrm{~mm}$ in diameter, which could produce thin filaments of the hydrogel capable of further manipulation. To further demonstrate the injectability of the hydrogel, the process of injection is shown in Figure S2. Based on these results, it was clear that the hydrogel could be continuously injected through a 0.5-mm needle.

Furthermore, the self-healing property of the hydrogel was confirmed by injection into the fresh wounds of SD rats with incisions on the dorsum panniculus carnosus. After subjecting the injections to a series of cuts, compressions, pinches, twists, and other external forces that could alter their shapes (Fig. 1d), the hydrogels were found to remain adhered to the wounds and were able to restore their original shapes while resisting the various external forces applied to the hydrogel-covered wounds, further validating the excellent properties of the hydrogel as a filler for diabetic skin wound treatment. Hydrogels typically have high viscosities; however, without a selfhealing ability, it would not be possible for the hydrogels to remain intact and free of cracks following the application of cutting and twisting forces. As shown in Fig. 1d, after removing the applied external force, our hydrogel could recover to its original appearance without any significant defects. Therefore, it is reasonable to conclude that the hydrogel had a good self-healing property.

Bacterial infection prevention is an advantageous quality that a self-healing hydrogel should offer to aid in faster wound repair. The composition of the crosslinked Ag-SHPEG hydrogels allows for intrinsic antibacterial properties that after further DFO integration, could efficiently advance the rate of wound healing. An agar diffusion test was used to assess this characteristic, in which the control, the hydrogel, and the DFO-hydrogel groups were individually plated on Staphylococcus aureus and observed for 1, 3 and 5 days (Fig. 2a) ${ }^{25}$. The inhibition zones of each group were measured on the final day, and it was found that the hydrogel and DFO-hydrogel plates produced inhibition zones of $(1.46 \pm 0.05 \mathrm{~mm})$ and $(2.05 \pm 0.08$ $\mathrm{mm}$ ), respectively (Fig. 2d). The hydrogel's antibacterial properties could be attributed to the reversible $\mathrm{Ag}-\mathrm{S}$ bond produced from crosslinking SH-PEG and $\mathrm{AgNO}_{3}$ and the hydrogel's interaction with sulfur-containing proteins in the bacterial cell membrane ${ }^{26,27}$. The enhanced prevention of bacterial infections for wound healing was evident in the DFO-hydrogel's measured inhibition zone, which was larger in comparison to that of the hydrogel. Because iron plays a critical role in the growth and metabolic activity of pathogenic microorganisms, the strong antibacterial properties indicated by the DFO-loaded hydrogels' inhibition zone were possibly attributed to iron chelation, which amplified the antibacterial effect of $\mathrm{Ag}^{+}$and is a promising indicator of the hydrogel's ability to prevent further bacterial infections from impeding the wound repair process or deteriorating wound conditions ${ }^{28}$.

Cell viability is essential to ensuring that the DFOloaded self-healing hydrogels neither induce cytotoxicity nor have detrimental effects on their target wounds. Figure $2 \mathrm{~b}$ shows the results of Live/Dead ${ }^{\star}$ stains added to the HUVECs and then imaged to assess the effects of the DFO-loaded hydrogels on cell viability ${ }^{29}$. The cells were cultured in three solutions: a blank solution for the control study, a solution with synthesized hydrogels, and a solution with DFO loaded into the hydrogels. The addition of the DFO-loaded and non-loaded hydrogels showed minute differences compared to the control with regard to hindering cell proliferation during the 3 days over which the samples were collected. HUVEC shape, which was observed to be a mixture of elongated fusiforms and filopodia, was also consistent throughout each group. This was further verified by using CCK-8, which enabled quantification of cytotoxicity and cell metabolic activity ${ }^{30}$. As shown in Fig. 2e, no significant difference was 


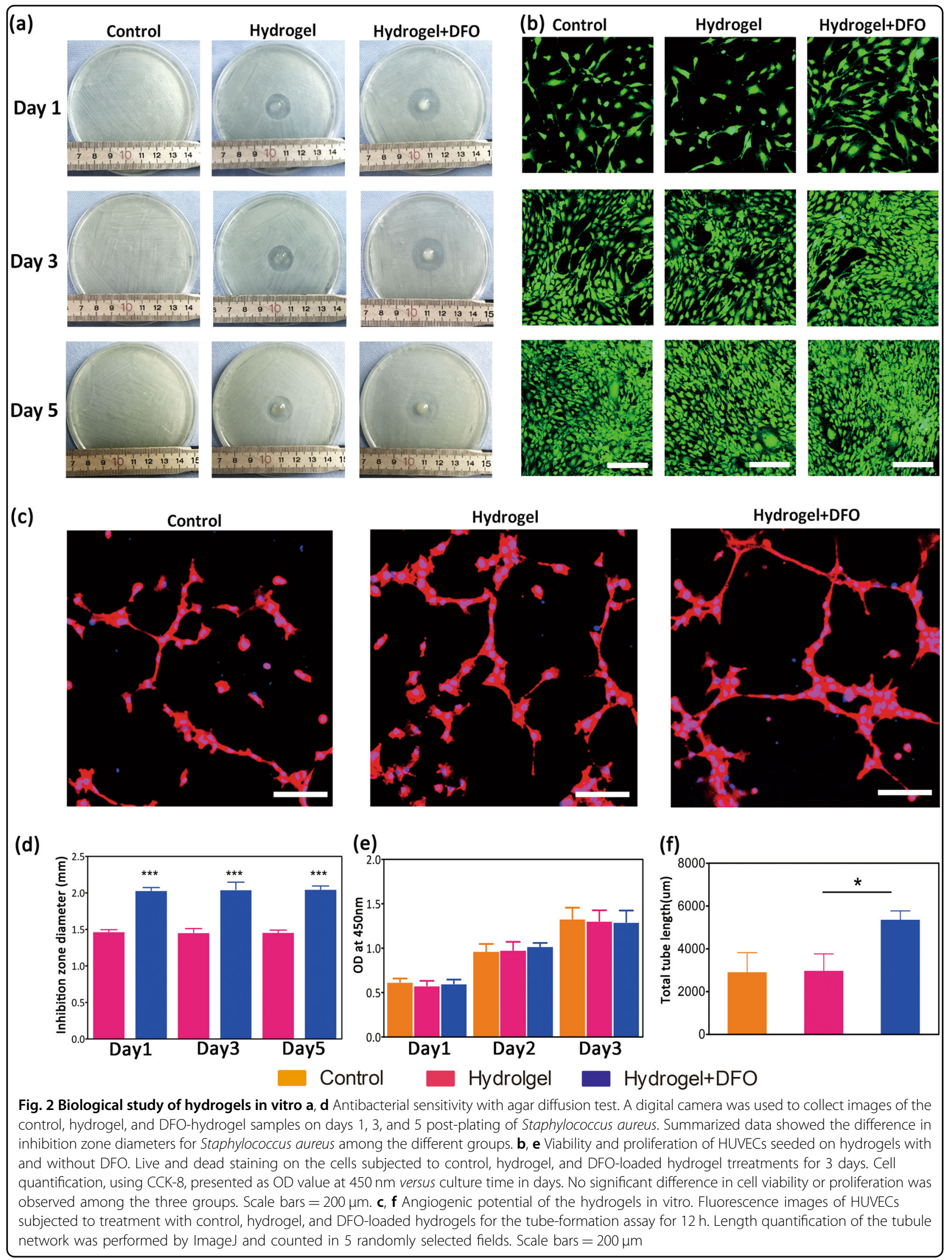




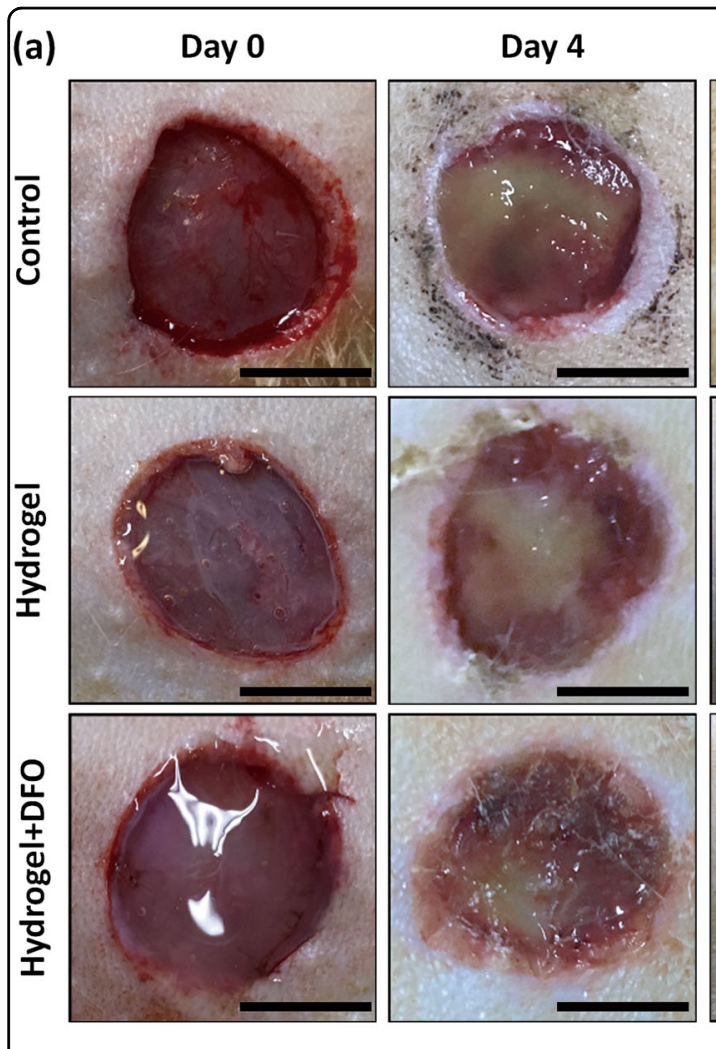

(b)

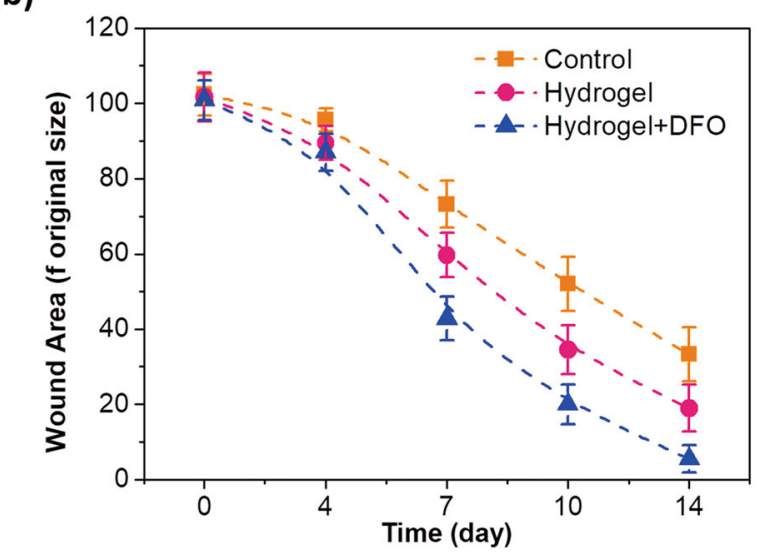

Day 7
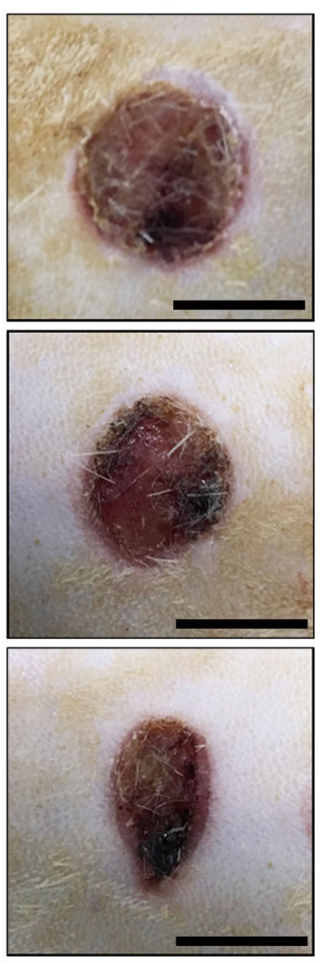

(c)
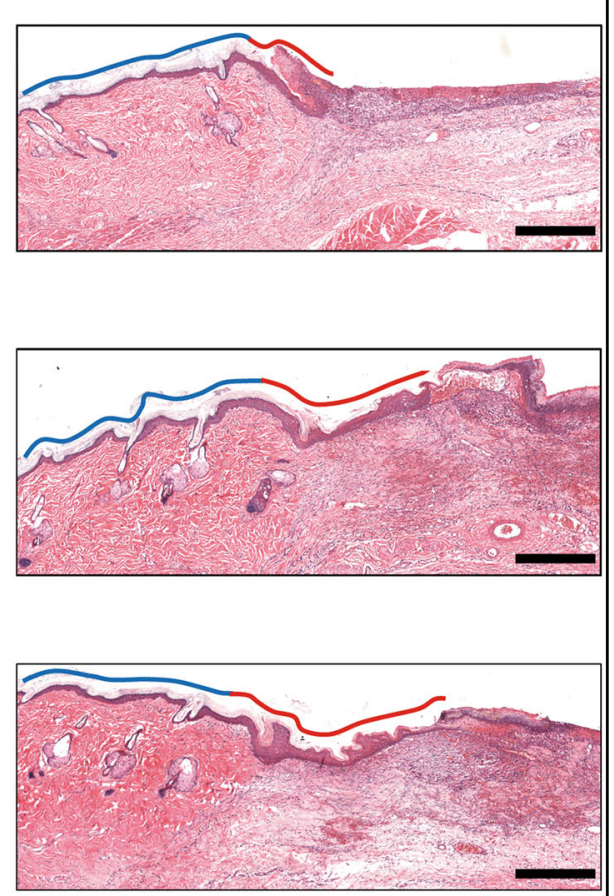

(d)

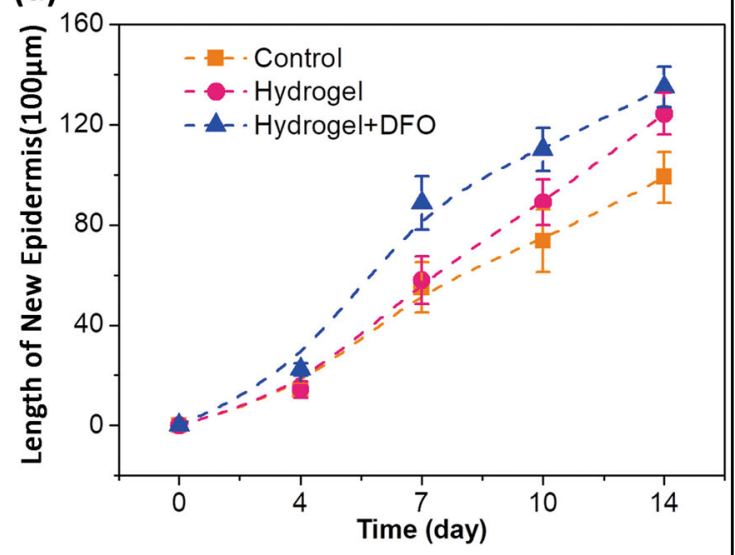

Fig. 3 Wound healing studies of SD rats injected with DFO-hydrogels in vivo. a A digital camera was used to collect images of wounds injected with the control, the hydrogel, and the DFO-hydrogel on 0, 4, 7, 10, and 14 days post-injection. Scale bars $=1 \mathrm{~cm}$. $\mathbf{b}$ Quantification of wound closure calculated as percent area relative to the original size with time. $\mathbf{c}$ Images of H\&E-stained full-thickness wounds, captured digitally with a microscope to observe the growth rate of the new epithelial layer in each group. Blue line: normal epidermis; Red line: new epidermis. Scale bars $=5 \mathrm{~mm}$. d Quantitative analysis of the new epidermis layer thicknesses using ImageJ

observed among the groups, and the optical density (OD) values increased with time. The results suggested that the HUVECs seeded on the hydrogels were able to proliferate during the course. The cell viability and proliferation results demonstrated that the hydrogels, regardless of the presence of DFO, were biocompatible with HUVECs.

Angiogenesis, the process of blood vessel formation, is an integral component of wound healing ${ }^{31}$. Ideally, the drug-loaded hydrogels should not only prevent bacterial infections but also promote angiogenesis and thus enhance the wound repair process. Fluorescence images and a tube-formation assay of the HUVECs cultivated on the three hydrogel groups were documented, as shown in Fig. 2c, to analyze whether the hydrogels could enhance angiogenesis of the cells. The HUVECs treated with DFOloaded hydrogels showed more comprehensive vascular 
tubule formations, with longer tube lengths in comparison to the control and hydrogel groups. This finding was attributed to the effects of the angiogenic drug DFO, which has been shown to increase the expression levels of ANGPT-2, EGF, PDGF-BB, and VEGF during treatment and thus can induce neovascularization ${ }^{32}$. Tube formation was also observed in both the control and hydrogel groups, but the tube structures appeared more fragile compared to their DFO-loaded counterparts. The total length of the formed tubes was counted in each group, and significant differences were observed between the DFO-hydrogels and the control and hydrogel groups (Fig. 2f), indicating that the hydrogels loaded with DFO were angiogenesis-inducing and thus may be more efficient in enhancing wound repairs in vivo.

SD rat models were subjected to full-thickness wounds inflicted by incision on the dorsum panniculus carnosus. The three treatments, which consisted of the control, the hydrogel, and the DFO-loaded hydrogel, were injected into the fresh wounds. The three groups were examined over the course of $0,4,7,10$ and 14 days for evidence of their wound healing tendencies based on images captured with a digital camera and a quantitative wound diameter analysis with ImageJ (Fig. 3a). The hydrogel-treated wounds were observed to have smaller wound diameters by day 7, especially in the DFO-hydrogel-injected wounds, which appeared dryer and had decreased by $50 \%$ of the original puncture size. The dryness indicated that excellent tissue reconstruction properties were presented early with advanced wound repair and that external bacterial infections had been prevented. In comparison, the wound sizes of both the control and hydrogel groups had only decreased by $30 \%$ of the original puncture size by day 7 . The wounds in the DFO-hydrogel group were nearly closed by day 14 (Fig. 3b). When the wound areas were statistically compared, it was apparent that the wound diameters in the first 2-3 days after puncture were very similar, which could be accounted for by the initiation of vessel formation and the lack of epithelial cells in the inflammatory stage (i.e., the healing stage that the wounds were in). However, the rate of wound closure was accelerated after day 7 post-injection and continued until day 14, at which point it was evident that the differences between the DFO-hydrogel and the two other groups were significant in terms of wound area, indicating that the hydrogel injected with DFO had good biocompatibility and provided support for tissue ingrowth.

A histological analysis was performed to contrast the rate of healing in the wound repair processes of the different treatment groups ${ }^{33}$. Histological cross-sections of the wounds were taken to observe the crawling distance of the new epidermis at each time point of harvest (Fig. 3c), followed by a quantitative analysis of the histological sections (Fig. 3d). Shown in Fig. 3c were the wound beds stained with $H \& E$ on day 7 post-injection. The hydrogel group appeared conducive to granulation tissue formation and exhibited a significantly faster epithelial crawling effect in comparison to that of the control group. However, the DFO-hydrogel group had more visible capillaries on the wound bed and a thicker and more defined granulation tissue structure, which served overall to enhance the re-epithelialization process more effectively than that of the other two groups. A statistical analysis was also undertaken, in which the lengths of the new epidermis layer were measured on days $0,4,7,10$ and 14 following the injection of the three groups. The differences were clearly visible, identified as a slightly longer epidermis in the DFO-hydrogel group compared to the hydrogel group, and a significantly longer epidermis in comparison to the control group, indicating that the DFO-hydrogels had a greater potential to support wound healing compared to the non-DFO-loaded hydrogels.

The prevention of bacterial infections from impeding the wound repair process or deteriorating wound conditions is important for wound healing. We have demonstrated that our DFO-loaded hydrogels had strong antibacterial properties in vitro. Furthermore, we carried out wound healing studies of the SD rat model injected with DFO-hydrogels to illustrate the antibacterial effects of this substrate in vivo. To visualize and localize the infection on the skin sections, an immunofluorescent stain was performed with anti-Staphylococcus aureus ${ }^{34}$. The result showed Staphylococcus aureus (green) and DAPI counterstains (nuclei; blue) depicting bacterial infection underneath the epidermal layer and underlying dermis (Fig. 4a). In comparison to the control group (right), the Staphylococcus aureus intensity was much lower in the hydrogel group, and the DFO-hydrogel had the least amount of bacteria present among the three groups (Fig. 4c). This finding indicated that the hydrogels had an antibacterial effect in vivo and that loading with DFO could further enhance the antibacterial effect of the hydrogels. Due to DFO's properties as an iron chelator, in which its binding of free iron ions decreases the concentration of iron, while simultaneously enhancing levels of hypoxia inducible factor 1- alpha (HIF-1 $\alpha$ ) and Vascular endothelial growth factor (VEGF) expression subsequently inducing neovascularization and antibacterial properties, we concluded that the hydrogels loaded with DFO may be more efficient for improving wound repairs ${ }^{5,35}$.

To further evaluate the mechanisms of hydrogel treatment on wound healing, the vascular endothelial-specific marker CD31 was stained for vascular endothelial cells treated by the three groups (Fig. 4b) ${ }^{34}$. The merged images of DAPI and CD31 showed that the DFO-hydrogels exhibited significantly increased 

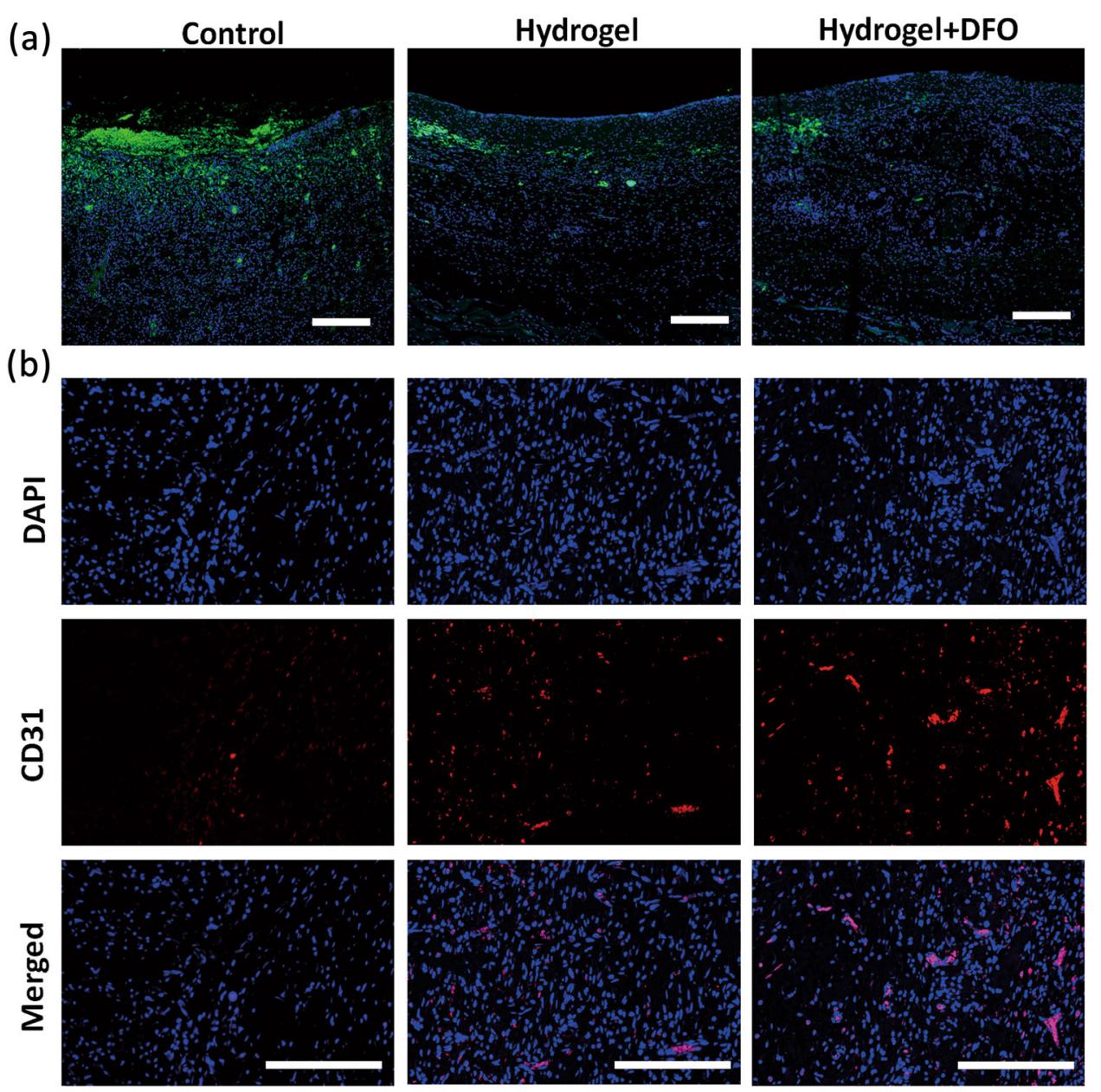

(c)

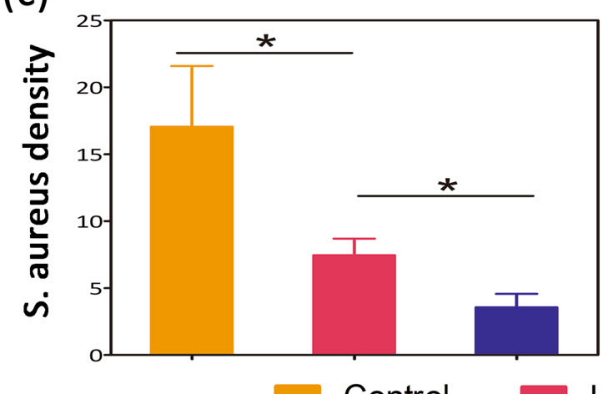

(d)

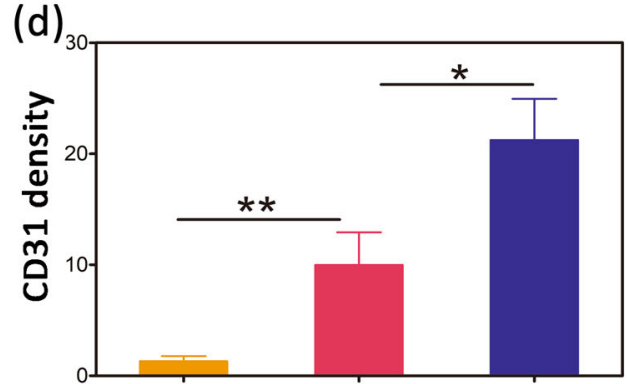

Control

Hydrolgel

Hydrogel+DFO

Fig. 4 Immunofluorescent stains in vivo a, c Wound healing studies of SD rat models injected with DFO-hydrogels to observe antibacterial effects in vivo. Representative images showing immunofluorescent stains with Staphylococcus aureus antibodies (green) and DAPI counterstaining (nucleus, blue). The summarized data showed the differences in the staining intensities of Staphylococcus aureus among the different groups. Scale bars $=100$ $\mu \mathrm{m}$. b, d Histological changes of wound healing in the DFO-hydrogel-injected wounds. Immunofluorescence staining of vascular endothelial cells treated with DFO-hydrogels. The cells were stained with CD31, and the nuclei were counterstained with DAPI. The summarized data showed the differences of the staining intensities of CD31 among the different groups. Scale bars $=100 \mu \mathrm{m}$

neovascularization based on a high level of CD31 expression. In contrast, the hydrogel alone displayed minimal CD31 expression, confirming that the DFOhydrogels provided an excellent 3D microenvironment scaffold for blood vessel formation (Fig. 4d).

\section{Conclusions}

In summary, we have described a multifunctional hydrogel scaffold with injectable, self-healing, antibacterial, and angiogenic properties for diabetic wound regeneration. The multifunctional hydrogel was 
prepared by the coordinative crosslinking of multi-arm thiolated PEG with $\mathrm{Ag}^{+}$accompanied by the loading of the angiogenic drug DFO. In vitro experiments confirmed the multifunctionality of the dynamic coordinative hydrogel, including good flexible manipulation, resistance to mechanical irritation, antibacterial properties, and angiogenic abilities. In vivo studies further demonstrated that the hydrogel could be injected to efficiently repair diabetic skin wounds with low incidence of bacterial infection and enhanced angiogenic activity. We anticipate that such a dynamic multifunctional hydrogel would show great promise in the regeneration of not only diabetic skin wounds but also different types of exposed wounds under disturbed physiological functions with a high risk of bacterial infection, and when exposed to external mechanical irritations.

\section{Acknowledgements}

H.C., R.C. and X.Z. contributed equally to this work. This work was financially supported by the National Key Research and Development Program of China (2018YFC1 106200 and 2018YFC1106204), National Natural Science Foundation of China (81702125, 21875092, 81572099, 51873107, and 21574091), the "Six Talent Peaks" program of Jiangsu Province (2018-XCL-013), the Science and Technology Commission of Shanghai Municipality (18ZR1434200), the Shanghai Sailing Program (17YF1411300), the Shanghai Municipal Education Commission-Gaofeng Clinical Medicine Grant Support (20171906), the Excellent Youth Scholars of Shanghai Jiao Tong University School of Medicine (17XJ11004), the Natural Science Foundation of Jiangsu Province (No. BK20160056), and the start-up fund (1-ZE7S) and central research fund (G-YBWS) from the Hong Kong Polytechnic University.

\section{Author details \\ 'Shanghai Key Laboratory for Prevention and Treatment of Bone and Joint Diseases, Shanghai Institute of Traumatology and Orthopaedics, Ruijin Hospital, Shanghai Jiao Tong University School of Medicine, 197 Ruijin 2nd Road, 200025 Shanghai, China. ${ }^{2}$ Department of Spinal Surgery, Renji Hospital, Shanghai Jiao Tong University School of Medicine, 160 Pujian Road, 200120 Shanghai, China. ${ }^{3}$ The Department of Biomedical Engineering, Hong Kong Polytechnic University, Hung Hom 999077 Hong Kong SAR, China. ${ }^{4}$ Loomis Chaffee School, 4 Batchelder Road, Windsor, Connecticut 06095, USA. ${ }^{5}$ Division of Engineering in Medicine, Department of Medicine, Brigham and Women's Hospital, Harvard Medical School, Cambridge, MA 02139, USA. Institute for Advanced Materials, School of Materials Science and Engineering, Jiangsu University, 212013 Zhenjiang, Jiangsu, China}

\section{Conflict of interest}

The authors declare that they have no conflict of interest.

\section{Publisher's note}

Springer Nature remains neutral with regard to jurisdictional claims in published maps and institutional affiliations.

Supplementary information is available for this paper at https://doi.org/ 10.1038/s41427-018-0103-9.

Received: 28 August 2018 Revised: 26 October 2018 Accepted: 4 November 2018.

Published online: 23 January 2019

\footnotetext{
References

1. Brem, H. \& Tomic-Canic, M. Cellular and molecular basis of wound healing in diabetes. J. Clin. Invest. 117, 1219-1222 (2007).

2. Okonkwo, U. \& DiPietro, L. Diabetes and wound angiogenesis. Int. J. Mol. Sci. 18, 1419 (2017)
}

3. Chellappan, D. K., Yenese, Y., Wei, C. C. \& Gupta, G. Nanotechnology and diabetic wound healing: a review. Endocr. Metab. Immune Disord. Drug. Targets 17, 87-95 (2017).

4. Chen, H. et al. Upregulating Hif-1alpha by hydrogel nanofibrous scaffolds for rapidly recruiting angiogenesis relative cells in diabetic wound. Adv. Health. Mater. 5, 907-918 (2016).

5. Chen, H. et al. Quickly promoting angiogenesis by using a DFO-loaded photocrosslinked gelatin hydrogel for diabetic skin regeneration. J. Mater. Chem. B 4, 3770-3781 (2016)

6. Adams, C. A. J. \& Deitch, E. A. Diabetic foot infections. (Zuckschwerdt, Munich, 2001).

7. Hobizal K. B., Wukich D. K. Diabetic foot infections: current concept review. Diabet Foot Ankle 3, 18409 (2012).

8. Martinez, N. et al. Defects in early cell recruitment contribute to the increased susceptibility to respiratory Klebsiella pneumoniae infection in diabetic mice. Microbes Infect. 18, 649-655 (2016).

9. Delamaire, M. et al. Impaired leucocyte functions in diabetic patients. Diabet. Med. 14, 29-34 (1997)

10. Broussard, K. C. \& Powers, J. G. Wound dressings: selecting the most appropriate type. Am. J. Clin. Dermatol. 14, 449-459 (2013).

11. Norouzi, M., Boroujeni, S. M., Omidvarkordshouli, N. \& Soleimani, M. Advances in skin regeneration: application of electrospun scaffolds. Adv. Healthc. Mater. 4, 1114-1133 (2015).

12. Casuso, P. et al. Injectable and self-healing dynamic hydrogels based on metal (I)-thiolate/disulfide exchange as biomaterials with tunable mechanical properties. Biomacromolecules 16, 3552-3561 (2015).

13. Lu, S. et al. Injectable and self-healing carbohydrate-based hydrogel for cell encapsulation. ACS Appl. Mater. Interfaces 7, 13029-13037 (2015).

14. Duscher, D. et al. Transdermal deferoxamine prevents pressure-induced diabetic ulcers. Proc. Natl Acad. Sci. USA 112, 94-99 (2015).

15. Kumar, V. A. et al. Highly angiogenic peptide nanofibers. ACS Nano 9, 860-868 (2015).

16. Hsieh, F.-Y., Tao, L., Wei, Y. \& Hsu, S.-h A novel biodegradable self-healing hydrogel to induce blood capillary formation. NPG Asia Mater. 9, e363 (2017).

17. Li, L. et al. Injectable self-healing hydrogel with antimicrobial and antifouling properties. ACS Appl. Mater. Interfaces 9, 9221-9225 (2017).

18. Liu, L. et al. A versatile dynamic mussel-inspired biointerface: from specific cell behavior modulation to selective cell isolation. Angew. Chem. Int. Ed. Engl. 57, 7878-7882 (2018)

19. Volkan, Y. et al. Mixed reversible covalent crosslink kinetics enable precise, hierarchical mechanical tuning of hydrogel networks. Adv. Mater. 29, 1605947 (2017).

20. Pan, G. et al. Dynamic introduction of cell adhesive factor via reversible multicovalent phenylboronic acid/cis-diol polymeric complexes. J. Am. Chem. Soc. 136, 6203-6206 (2014).

21. Xiaozhong, Q. \& Zhenzhong, Y. Benzoic-imine-based physiological-pHresponsive materials for biomedical applications. Chem. Asian J. 11, 2633-2641 (2016).

22. Xiaowei, Z. et al. A drug-self-gated mesoporous antitumor nanoplatform based on pH-sensitive dynamic covalent bond. Adv. Funct. Mater. 27, 1605985 (2017).

23. Xing, R. et al. An injectable self-assembling collagen-gold hybrid hydrogel for combinatorial antitumor photothermal/photodynamic therapy. Adv. Mater. 28, 3669-3676 (2016)

24. Dhar, P., Katiyar, A. \& Maganti, L. S. Smart viscoelastic and self-healing characteristics of graphene nano-gels. J. Appl. Phys. 120, 214304 (2016).

25. Runrun, W. et al. Spatio-design of multidimensional Prickly Zn-doped CuO nanoparticle for efficient bacterial killing. Adv. Mater. Interfaces 3, 1600472 (2016).

26. Juby, K. A. et al. Silver nanoparticle-loaded PVA gum acacia hydrogel: synthesis, characterization and antibacterial study. Carbohydr. Polym. 89, 906-913 (2012).

27. Aduba, D. C. Jr et al. Fabrication, characterization, and in vitro evaluation of silver-containing arabinoxylan foams as antimicrobial wound dressing. J. Biomed. Mater. Res. A. 104, 2456-2465 (2016).

28. Anushila, C. \& O'Brian, M. R. Rapid evolution of a bacterial iron acquisition system. Mol. Microbiol. 108, 90-100 (2018).

29. Shucheng, L. et al. Dynamically PEGylated and borate-coordination-polymercoated polydopamine nanoparticles for synergetic tumor-targeted, chemophotothermal combination therapy. Small 14, 1703968 (2018). 
30. Pan, G. et al. Biomimetic design of mussel-derived bioactive peptides for dualfunctionalization of titanium-based biomaterials. J. Am. Chem. Soc. 138 15078-15086 (2016)

31. Botusan, I. R. et al. Stabilization of HIF-1alpha is critical to improve wound healing in diabetic mice. Proc. Natl Acad. Sci. USA 105, 19426-19431 (2008)

32. Qiu, M. et al. Angiogenic and osteogenic coupling effects of deferoxamineloaded poly(lactide-co-glycolide)-poly(ethylene glycol)-poly(lactide-co-glycolide) nanoparticles. Appl. Sci. 6, 290 (2016).
33. Hou, Z., Nie, C., Si, Z. \& Ma, Y. Deferoxamine enhances neovascularization and accelerates wound healing in diabetic rats via the accumulation of hypoxia-inducible factor-1a. Diabetes Res. Clin. Pract. 101, 62-71 (2013).

34. Soong, G. et al. Staphylococcus aureus Protein A mediates invasion across airway epithelial cells through activation of RhoA GTPase signaling and proteolytic activity. J. Biol. Chem. 286, 35891-35898 (2011)

35. Moon, J. H., Kim, C., Lee, H. S., Kim, S. W. \& Lee, J. Y. Antibacterial and antibiofilm effects of iron chelators against Prevotella intermedia. J. Med. Microbiol. 62(Pt 9), 1307-1316 (2013). 